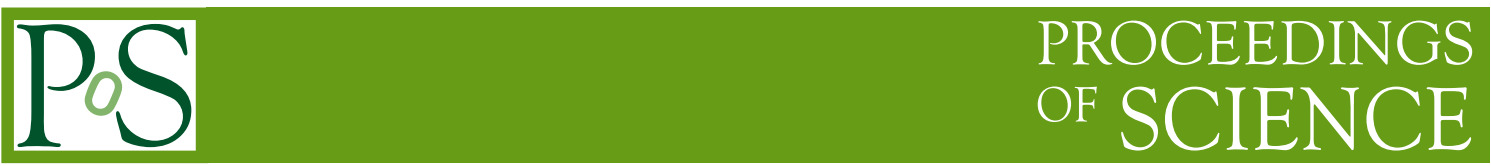

\title{
Recent diboson and multiboson results in ATLAS
}

Markus Cristinziani*i

On behalf of the ATLAS Collaboration

Physikalisches Institut, Universität Bonn, Nussallee 12, 53115 Bonn, Germany.

E-mail: cristinz@uni-bonn.de

\begin{abstract}
Recent measurements of the associated production of two or three massive vector bosons in proton-proton collisions at the Large Hadron Collider, collected by the ATLAS detector at a centre-of-mass energy of $\sqrt{s}=13 \mathrm{TeV}$, are reported. The diboson analyses target the production and decay modes $W W \rightarrow e v_{e} \mu v_{\mu}, W Z \rightarrow \ell^{\prime} v \ell \ell(\ell=e, \mu), Z Z \rightarrow \ell \ell v v$, and the $4 \ell$ final state. Fiducial inclusive cross sections are compared to state-of-the-art Standard Model calculations and several unfolded differential distributions are measured. In the $W Z$ channel, the helicities of the $W$ and $Z$ bosons are probed. Results are interpreted in terms of limits on anomalous gauge couplings in the framework of effective field theories. Finally, the first evidence for the production of three massive vector bosons $(W W W, W W Z, W Z Z)$ is presented.
\end{abstract}

7th Annual Conference on Large Hadron Collider Physics - LHCP2019

20-25 May, 2019

Puebla, Mexico

\footnotetext{
*Speaker.

${ }^{\dagger}$ Supported by the European Research Council grant ERC-CoG-617185 and by the German Federal Ministry of Education and Research (FSP-103)
} 


\section{Introduction}

Five recent measurements of the ATLAS Collaboration [1-5] are presented of the production of two or three massive vector bosons $V(V=W$ or $Z)$ in proton-proton $(p p)$ collisions at a centreof-mass energy of $\sqrt{s}=13 \mathrm{TeV}$ at the Large Hadron Collider (LHC). The diboson production measurements of $W W$ [1], $W Z$ [2], $Z Z$ [3], and $Z Z$ along with $Z \rightarrow 4 \ell$ and $H \rightarrow 4 \ell$ [4] have been performed using the data collected in 2015 and 2016, corresponding to a dataset of $36.1 \mathrm{fb}^{-1}$, while the measurement targeting the production of three bosons, $W W W, W W Z, W Z Z$ [5], are based on a larger dataset of $79.8 \mathrm{fb}^{-1}$, i.e. including 2017 data.

The study of the production of multiple massive vector bosons through interactions of quarks and gluons probes the electroweak (EW) non-Abelian gauge structure of the Standard Model (SM) and allows further tests of the strong interaction between quarks and gluons. It provides the means to directly probe triple (TGC) and quartic (QGC) gauge boson couplings. On the other hand, a broad range of new phenomena beyond the SM (BSM) are predicted to reveal themselves through multiboson production. Improved constraints from precise measurements can potentially probe scales of new physics in the multi-TeV range and provide a way to look for signals of new physics in a model-independent way.

Sizeable production cross sections combined with the large sample of $p p$ collision data delivered by the LHC, enable these processes to be studied with a better statistical precision than was possible in previous measurements or establish them for the first time. While $W$ bosons are produced more copiously than $Z$ bosons, the signal-to-background ratio is in general more advantageous in channels containing leptonically-decaying $Z$ bosons and therefore the final states and analyses have similar sensitivities to uncover departures from the SM predictions.

\section{Measurements of two massive vector bosons using 2015 and 2016 data}

\subsection{Production of $W W \rightarrow e v_{e} \mu v_{\mu}$}

The cross section for the production of $W^{+} W^{-}$pairs is measured in a fiducial phase space selecting one electron and one muon, targeting the decay $W W \rightarrow e^{ \pm} v \mu^{\mp} v$. The fiducial phase space is chosen to be orthogonal to the ATLAS $H \rightarrow W W$ measurements by means of a requirement on the dilepton invariant mass. Events with a same-flavour lepton pair or with jets with a transverse momentum $\left(p_{\mathrm{T}}\right)$ above $35 \mathrm{GeV}$ are discarded because they have a larger background from the Drell-Yan process, or from top quarks, respectively. Additional requirements are imposed in order to reduce the dominant backgrounds. After event selection, the signal-to-background ratio is approximately 2, with top-quark being the largest background, followed by Drell-Yan, $W+$ jets and $W Z$ events. The systematic uncertainty for the inclusive cross-section measurement amounts to $7 \%$, dominated by the uncertainties related to the identification of $b$-quark-initiated jets ( $b$-tag), the estimation of the $W+$ jets background, and the uncertainties related to the identification of jets.

The measured fiducial cross section is found to be consistent with theoretical predictions, including next-to-next-to leading order (NNLO) QCD and next-to leading order (NLO) EW corrections (Figure 1a). The result is: $\sigma_{W W \rightarrow e v_{e} \mu v_{\mu}}^{\text {fid. }}=379.1 \pm 5.0$ (stat.) \pm 25.4 (syst.) \pm 8.0 (lumi.) fb. The fiducial cross section is also measured as a function of the $p_{\mathrm{T}}$ threshold for the jet veto, where the fiducial cross section rises by about $30 \%$ when accepting events containing jets with a $p_{\mathrm{T}}$ of 
up to $60 \mathrm{GeV}$, as compared with $30 \mathrm{GeV}$. Predictions agree within uncertainties with the data, but are consistently at the lower bound of these and a slight slope in the ratio of predictions to data is observed.

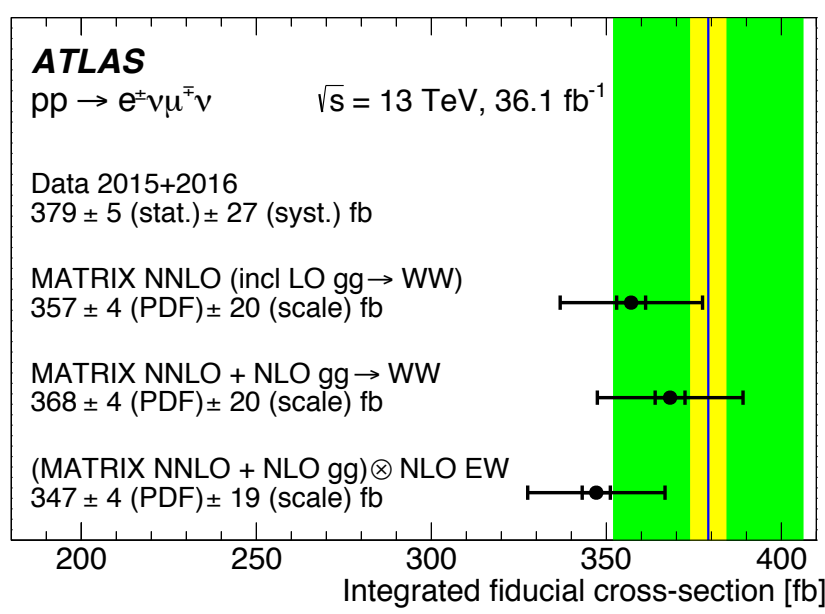

(a)

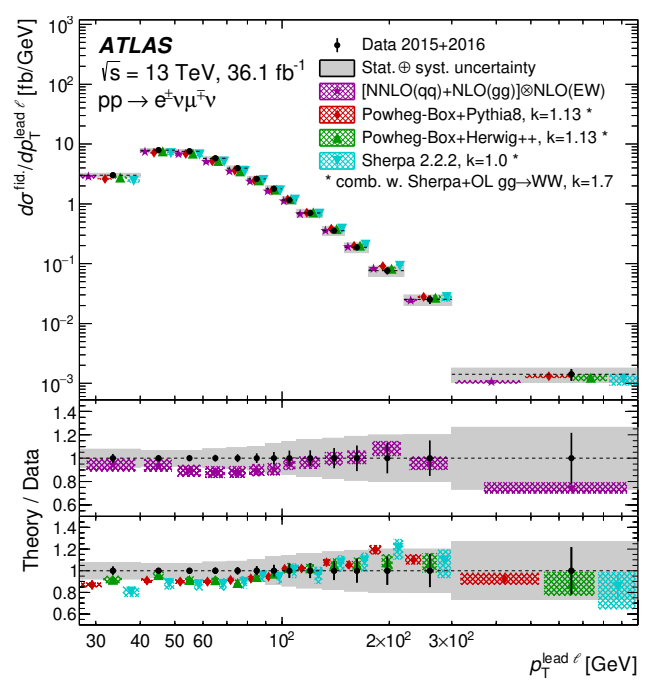

(b)

Figure 1: (a) Comparison of the measured fiducial cross section, indicated by a vertical line and bands, with various theoretical predictions, indicated as points with error bars [1]. (b) Measured fiducial cross section as a function of $p_{\mathrm{T}}^{\text {lead } \ell}$, compared to corrected NNLO predictions and various Monte-Carlo simulations [1]. Theoretical uncertainties correspond to PDF+scale variations.

Six differential distributions involving kinematic variables of the final-state leptons are measured. Three of them characterise the energy of the process: the transverse momentum of the leading lepton $p_{\mathrm{T}}^{\text {lead } \ell}$, the invariant mass $m_{e \mu}$ and the transverse momentum of the dilepton system. Three further distributions probe angular correlations and the spin state of the $W W$ system: the rapidity of the dilepton system, the difference in azimuthal angle between the decay leptons $\Delta \phi_{e \mu}$, and $\left|\tanh \left(\left(\Delta \eta_{e \mu}\right) / 2\right)\right|$, where $\Delta \eta_{e \mu}$ is the difference between the pseudorapidities of the leptons. The differential cross sections are compared with several predictions from perturbative QCD calculations. Data and theory show fair agreement for all distributions, except for low values of $m_{e \mu}$, for $\Delta \phi_{e \mu}<1.8$, as well as for low values of $p_{\mathrm{T}}^{\text {lead } \ell}$ (Figure 1b).

\subsection{Production of $W Z \rightarrow \ell^{\prime} v \ell \ell$}

The $W Z$ production cross section is measured within a fiducial phase space closely matching the detector acceptance, both inclusively and differentially as a function of several individual variables related to the kinematics of the $W Z$ system and to the jet activity in the event. The $W$ and $Z$ bosons are reconstructed using their decay modes into electrons or muons, employing the so-called resonant-shape algorithm. All final states with three leptons and missing transverse momentum $\left(E_{\mathrm{T}}^{\mathrm{miss}}\right)$ are considered, and categorised into the channels eee, $\mu e e, e \mu \mu$ and $\mu \mu \mu$, where the first lepton is the one assigned to the $W$ boson decay. No requirement on the number of jets is applied. After event selection, the signal-to-background ratio is approximately 4 and the dominant back- 
grounds are processes with misidentified leptons and $Z Z$ production. Systematic uncertainties are considerably reduced by combining the different final states. The dominant uncertainty is related to the estimation of the contribution by the misidentified leptons.

The measured inclusive cross section in the fiducial region is $\sigma_{W^{ \pm} Z \rightarrow \ell^{\prime} v \ell \ell}^{\text {fid. }}=63.7 \pm 1.0$ (stat.) \pm 2.3 (syst.) \pm 1.4 (lumi.) fb, in agreement with the NNLO Standard Model expectation of $61.5_{-1.3}^{+1.4} \mathrm{fb}$. The ratio of the $W^{+} Z$ cross section to the $W^{-} Z$ cross section, which is sensitive to the parton distribution functions (PDF), is also measured. Here, most of the systematic uncertainties almost cancel out in the ratio. The result, $\sigma_{W^{+} Z}^{\text {fid. }} / \sigma_{W^{-} Z}^{\text {fid. }}=1.47 \pm 0.05$ (stat.) \pm 0.02 (syst.), is compared with the SM calculation and several PDF sets in Figure 2a.

The $W Z$ production cross section is measured as a function of several kinematic variables and compared with SM predictions at NNLO from the MATRIX calculation and at NLO from the Powheg+Pythia and Sherpa event generators: the transverse momentum of the $Z$ boson, the transverse momentum of the $W$ boson, the transverse mass of the $W Z$ system $m_{\mathrm{T}}^{W Z}$ (Figure $2 \mathrm{~b}$ ), the azimuthal angle between the $W$ and $Z$ bosons $\Delta \phi(W, Z)$, the $p_{\mathrm{T}}$ of the neutrino associated with the decay of the $W$ boson, and, finally, the absolute difference between the rapidities of the $Z$ boson and the lepton from the decay of the $W$ boson. The differential cross-section distributions are well described by the theory predictions, with the exception of the jet multiplicity. The MATRIX calculations show the best agreement with the data, for instance for the $\Delta \phi(W, Z)$ distribution which is sensitive to QCD higher-order perturbative effects.

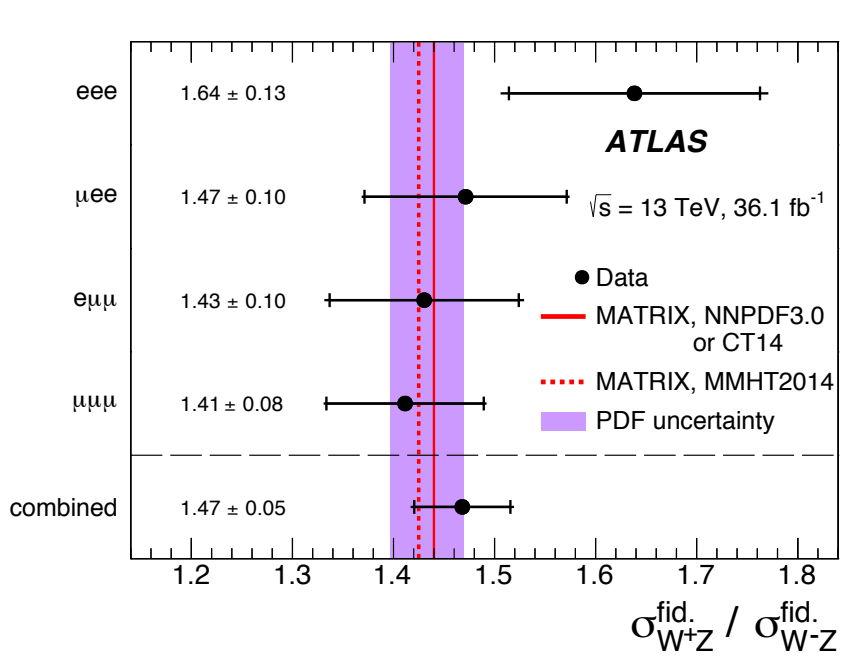

(a)

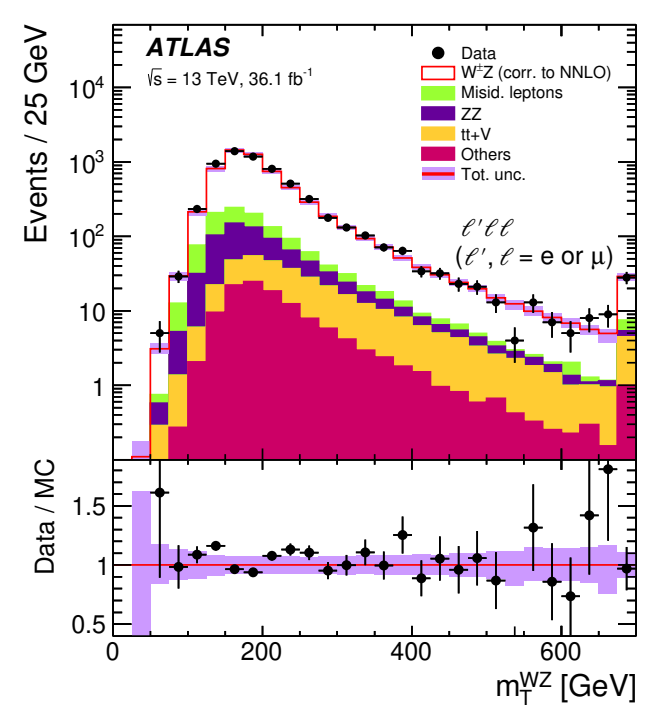

(b)

Figure 2: (a) Measured ratio $\sigma_{W^{+} Z}^{\text {fid. }} / \sigma_{W^{-} Z}^{\text {fid. }}$ of $W^{+} Z$ and $W^{-} Z$ integrated cross sections in the fiducial phase space in each of the four channels and for the combination [2]. (b) Distribution of the kinematic variable $m_{\mathrm{T}}^{W Z}$ [2].

An analysis of angular distributions of the leptons from the decays of the $W$ and $Z$ bosons has been performed. The normalised differential distribution as a function of $\cos \theta_{\ell, V}$ depends on the longitudinal $\left(f_{0}\right)$, transverse left-handed $\left(f_{L}\right)$ and transverse right-handed $\left(f_{R}\right)$ helicity fractions, where $\theta_{\ell, V}$ is defined using the helicity frame, as the decay angle of the lepton in the $V$ boson 
rest frame relative to the $V$ direction in the $W Z$ centre-of-mass frame. The existence of the longitudinally polarised state is a consequence of the non-vanishing mass of the bosons generated by the electroweak symmetry breaking (EWSB) mechanism. The measurement of the polarisation in diboson production therefore tests both the SM innermost gauge symmetry structure, through the existence of TGC, and the particular way this symmetry is spontaneously broken, via the longitudinal helicity state. Angular observables can be used to look for new interactions that can lead to different polarisation behaviour than predicted by the SM, to which the $W Z$ final state would be particularly sensitive.

Helicity fractions of pair-produced vector bosons are measured for the first time in hadronic collisions. The three helicity fractions of the $W$ and $Z$ bosons are measured using a template fit to the $\cos \theta_{\ell, V}$ distributions. Integrated over the fiducial region, the longitudinal polarisation fractions of the $W$ and $Z$ bosons in $W Z$ events are measured to be $f_{0}^{W}=0.26 \pm 0.06$ and $f_{0}^{Z}=0.24 \pm 0.04$, in agreement with the SM predictions at NLO in QCD and at leading order (LO) for EW corrections, of $0.238 \pm 0.003$ and $0.230 \pm 0.003$, respectively. The observed significances of the measurements are $4.2 \sigma$ and $6.5 \sigma$ for $f_{0}^{W}$ and $f_{0}^{Z}$, respectively. The measurements are dominated by statistical uncertainties.

The differences of the left and right transverse polarisations are also measured, with the constraint $f_{0}+f_{L}+f_{R}=1$. The values of $f_{0}$ and $f_{\mathrm{L}}-f_{\mathrm{R}}$, measured in $W Z$ events, are shown in Figure 3 . They agree with the predictions from PowHeg+PythIA and MATRIX within less than one and two standard deviations of their uncertainties for $f_{0}$ and $f_{\mathrm{L}}-f_{\mathrm{R}}$, respectively. No stringent constraints nor clear inconsistencies between measurements and predictions can be deduced. Polarisation measurements for each charge of the $W$ boson might be helpful in the investigation of $C P$ violation effects in the interaction between gauge bosons. In the longer term, measuring the scattering of longitudinally polarised vector bosons will be a fundamental test of EWSB.

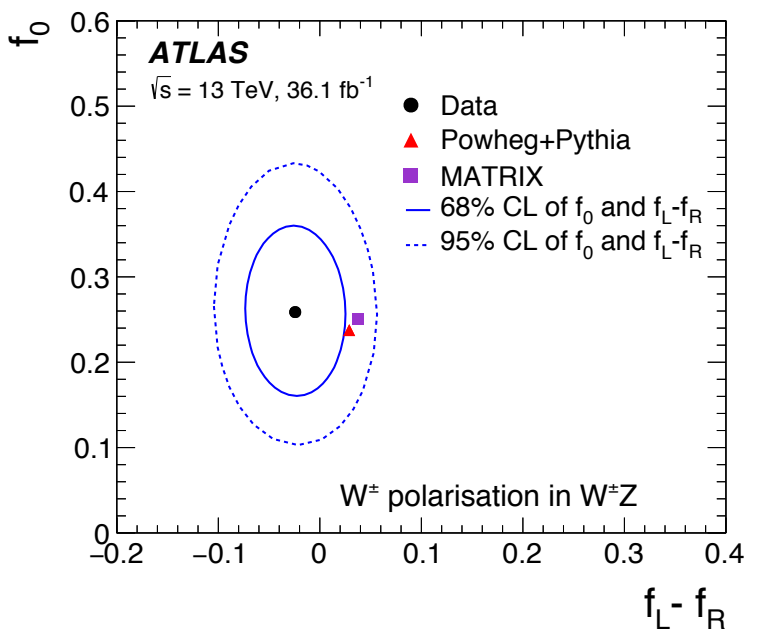

(a)

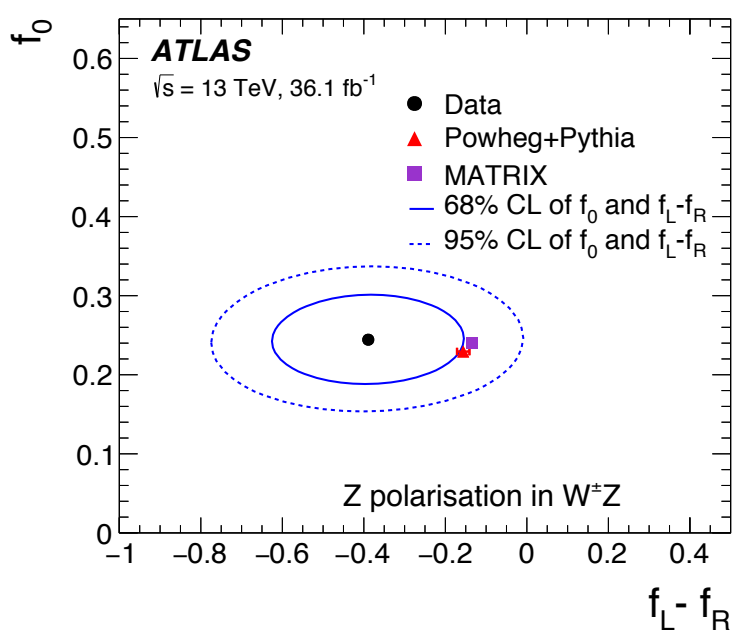

(b)

Figure 3: Measured helicity fractions $f_{0}$ and $f_{\mathrm{L}}-f_{\mathrm{R}}$ for the (a) $W$ boson and (b) $Z$ boson in $W^{ \pm} Z$ events, compared with predictions at LO for the electroweak interaction and with $\sin ^{2} \theta_{\mathrm{W}}=0.23152$ from PowHEg+PYTHIA and MATRIX [2]. 


\subsection{Production of $Z Z \rightarrow \ell \ell v v$}

The $Z Z$ production in the $\ell \ell v v$ final state has a larger branching fraction but suffers from higher background contamination in comparison with the $Z Z \rightarrow 4 \ell$ channel. To ensure a good signal-tobackground ratio, the experimental selection requires one $Z$ boson boosted against the other in the transverse plane, which results in a pair of high- $p_{\mathrm{T}}$ leptons and significant $E_{\mathrm{T}}^{\mathrm{miss}}$. The $\ell \ell v v$ channel thus offers higher data statistics than the $4 \ell$ channel for events with high- $p_{\mathrm{T}} Z$ bosons, and competitive precision for integrated and differential measurements, as well as good sensitivity to anomalous TGC (aTGC). The integrated cross section of $Z Z$ production is measured in a fiducial phase space and then extrapolated to a total phase space. The signal yield is determined through a fit to the observed $E_{\mathrm{T}}^{\text {miss }}$ spectrum (Figure $4 \mathrm{a}$ ), which leads to improved sensitivity compared with a simple event-counting method.

Candidate events are selected by requiring exactly two electrons or muons with opposite charges. The dilepton invariant mass is required to be compatible with the $Z$ boson mass. Candidate events are required to have significant $E_{\mathrm{T}}^{\text {miss }}$ and $V_{\mathrm{T}} / S_{\mathrm{T}}>0.65$, where $V_{\mathrm{T}}$ is the magnitude of the vector sum of transverse momenta of selected leptons and jets, and $S_{\mathrm{T}}$ is the scalar $p_{\mathrm{T}}$ sum of the corresponding objects. Additional selection criteria based on angular variables are imposed to ensure the desired detector signature. Finally, events containing one or more $b$-tagged jets are vetoed to further suppress the $t \bar{t}$ and $W t$ backgrounds. After the event selection, the overall signalto-background ratio is about 1.7. The $W Z$ and non-resonant- $\ell \ell$ backgrounds account for $72 \%$ and $21 \%$ of the total background contribution, respectively, and are estimated from control regions in data. Systematic uncertainties are dominated by the uncertainties in the background modelling followed by the uncertainties in the jet identification.

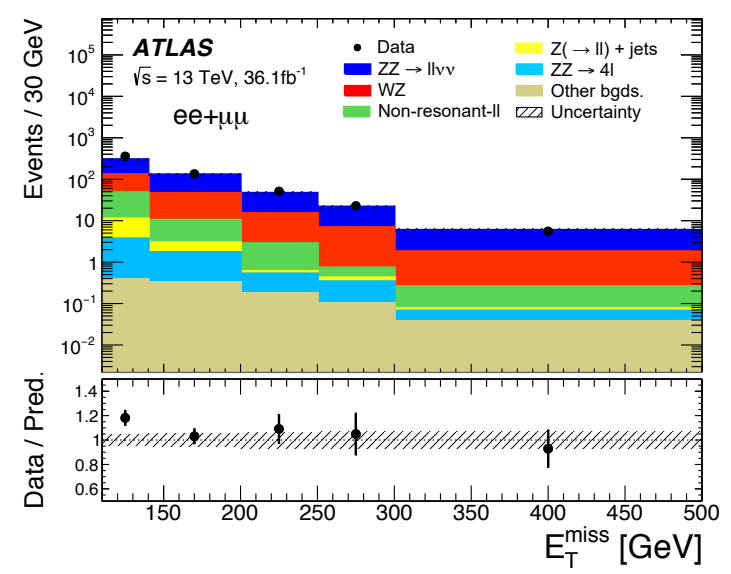

(a)

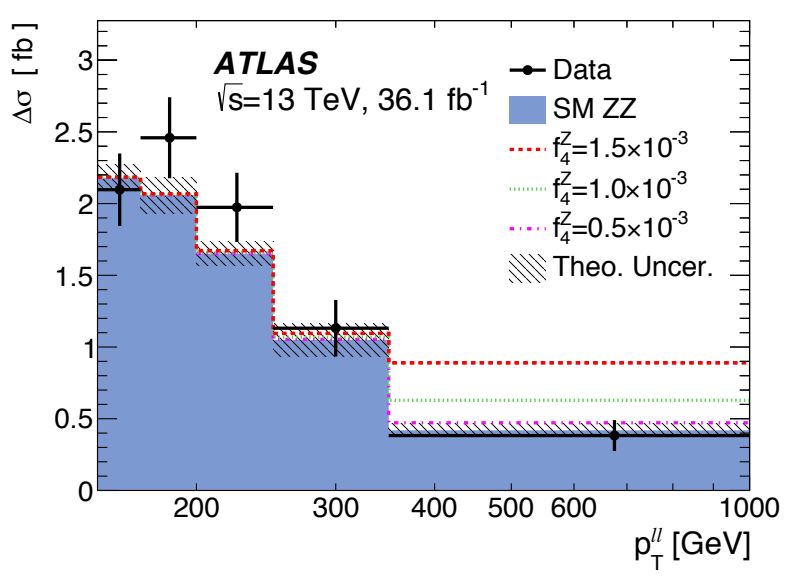

(b)

Figure 4: (a) Observed and expected $E_{\mathrm{T}}^{\text {miss }}$ distribution for the $Z Z$ analysis [3]. (b) Unfolded measured distribution of $p_{\mathrm{T}}^{\ell \ell}$, compared with the SM prediction from PowHEg to which are added possible aTGC contributions for different values of the strength of the coupling parameters (here for $f_{4}^{Z}$ ) [3].

The fiducial cross section of the combined $e e$ and $\mu \mu$ channels is measured to be $\sigma_{Z Z \rightarrow \ell \ell v v}^{\text {fid. }}=$ $25.4 \pm 1.4$ (stat.) \pm 0.9 (syst.) \pm 0.5 (lumi.) fb, in agreement with the SM prediction of $22.4 \pm 1.3 \mathrm{fb}$. 
The integrated cross sections in the total and fiducial phase spaces are measured with an uncertainty of $7 \%$, which is significantly better than the previous measurement using the $8 \mathrm{TeV}$ data. The measured cross sections is about $13 \%$ higher than the NNLO (quark-initiated) and NLO (gluoninitiated) SM predictions for $Z Z$ production, but the difference is not significant considering the measurement and prediction uncertainties.

Differential cross sections are reported in the fiducial region for eight kinematic variables, which are sensitive to effects from higher-order corrections and possible BSM physics. These variables include the transverse momenta of the leading lepton, of the leading jet, of the dilepton system ( $p_{\mathrm{T}}^{\ell \ell}$, Figure $4 \mathrm{~b}$ ), and of the $Z Z$ system; the transverse mass of the $Z Z$ system, the absolute rapidity of the dilepton system, the azimuthal angle difference between the two leptons, and the number of jets. Differential cross sections are reported for these eight kinematic variables in the fiducial phase space, and no significant deviation from the expectations is found.

\subsection{Four-lepton production}

In $p p$ collisions, four-lepton production is expected to receive contributions from several SM physics processes, resulting in a rich predicted cross-section spectrum, shown as a function of the invariant four-lepton mass $m_{4 \ell}$ in Figure 5.

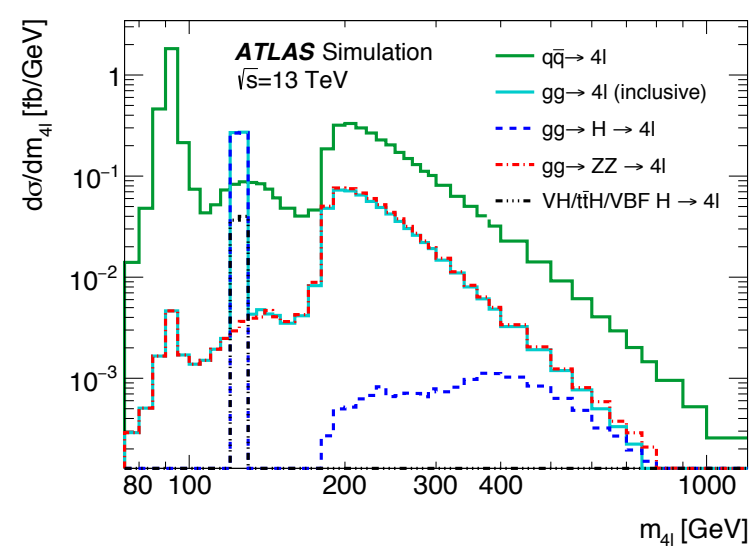

(a)

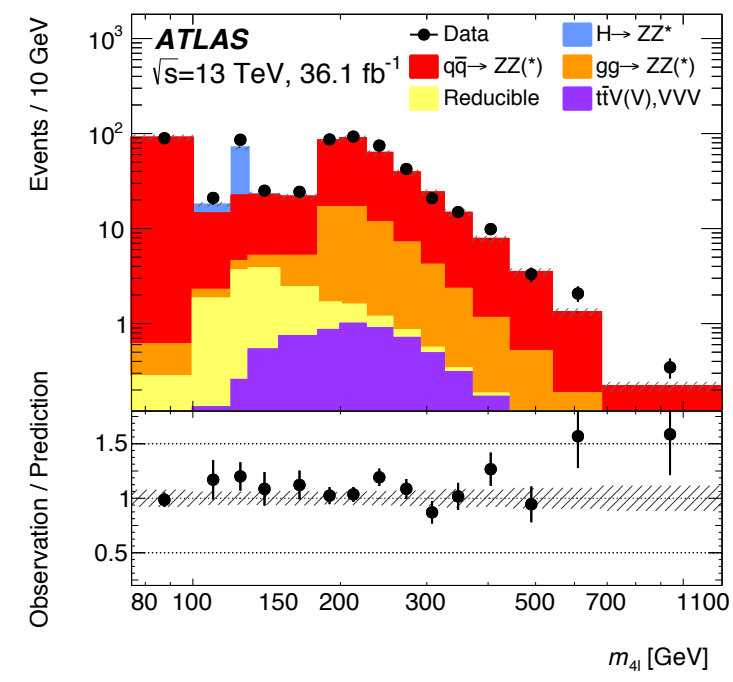

(b)

Figure 5: (a) Differential cross sections as a function of the four-lepton invariant mass $m_{4 \ell}$ predicted by simulation [4]. The total $g g \rightarrow 4 \ell$ includes contributions from $g g \rightarrow H^{(*)} \rightarrow 4 \ell$ as well as $g g \rightarrow 4 \ell$ and the interference between the two. The $q \bar{q} \rightarrow 4 \ell$ and $g g \rightarrow 4 \ell$ processes include off-shell Higgs boson production. (b) Selected events as a function of $m_{4 \ell}$ compared to the total SM prediction [4].

Largest in magnitude is the quark-induced $t$-channel process $q \bar{q} \rightarrow 4 \ell$, with leptonic decays of the $Z$ bosons. Gluon-induced $g g \rightarrow 4 \ell$ production also occurs, via an intermediate quark loop. The theoretical uncertainties in the SM prediction for this latter contribution are comparatively large. At around $m_{4 \ell} \simeq m_{Z}$, single resonant $Z \rightarrow 4 \ell$ production through QED radiative processes leads to a peak in the spectrum, and allows an extraction of the cross section and branching fraction for 
$Z \rightarrow 4 \ell$ to be made. Pairs of $Z$ bosons can also be produced from the decay of an intermediate Higgs boson. The majority of these are produced via gluon-gluon fusion, with minor contributions from vector-boson fusion and associated production with vector bosons or top-quark pairs. There is resonant production around the Higgs boson mass, as well as off-shell production at higher mass values, which is enhanced at approximately $350 \mathrm{GeV}$ due to top-quark loops in the gluon-gluon fusion mechanism. At around $180 \mathrm{GeV}$ there is an enhancement of all the processes involving two $Z$ bosons, as on-shell production is possible above this mass. The box diagram processes $g g \rightarrow 4 \ell$ and $g g \rightarrow H^{(*)} \rightarrow 4 \ell$ interfere destructively in the SM. While interference is maximal around $m_{4 \ell}=$ $220 \mathrm{GeV}$, the relative effect of the $g g \rightarrow H^{(*)} \rightarrow 4 \ell$ contribution to the overall $g g \rightarrow 4 \ell$ lineshape is most pronounced above $350 \mathrm{GeV}$, as is visible in Figure 5. The off-shell Higgs production rate may be affected by BSM processes involving additional heavy particles, or modifications of the Higgs couplings, even if there is no effect on on-shell Higgs boson production.

Events are required to contain two pairs of same-flavour opposite-sign (SFOS) leptons. Contributions from leptonically decaying $\tau$-leptons and quarkonia are reduced through requirements on the invariant masses of the dilepton pairs, but otherwise the selection is rather inclusive. The measurement is made differentially in the invariant mass $m_{4 \ell}$ of the four-lepton system, and doubledifferentially as a function of $m_{4 \ell}$ versus the transverse momentum of the four-lepton system $p_{\mathrm{T}}^{4 \ell}$, the rapidity of the system, the matrix-element discriminant $D_{\mathrm{ME}}$ designed to isolate off-shell Higgs boson contributions, and the final state lepton flavour channel. The $m_{4 \ell}$ measurement is also made separately for each flavour combination of leptons in the event: $4 e, 4 \mu$ and $2 e 2 \mu$. The doubledifferential cross sections can provide additional sensitivity to the various subprocesses contributing to the measured final state. For example, the $p_{\mathrm{T}}^{4 \ell}$ is expected to discriminate $g g \rightarrow Z Z$ from $q \bar{q} \rightarrow Z Z$. They are also of interest for future interpretations, as some BSM contributions can have an impact which depends upon the final-state lepton flavours.

The limiting source of uncertainty in this measurement is the statistical uncertainty, which is many times larger than the total systematic uncertainty in some bins. Experimental and theoretical sources both contribute to the systematic uncertainty, and their relative impact varies depending on the bin. The main systematic uncertainties are due to the lepton identification and uncertainties related to the luminosity and pile-up. The measurements are consistent with the predictions of the SM. The signal strength of the gluon-gluon fusion production process is measured to be $\sigma_{g g \rightarrow 4 \ell} / \sigma_{g g \rightarrow 4 \ell}^{\mathrm{SM}}=1.3 \pm 0.5$ compared to an expected value of $1.0 \pm 0.4$. A value for the $Z \rightarrow 4 \ell$ branching fraction of $(4.70 \pm 0.32$ (stat.) \pm 0.25 (syst. $)) \times 10^{-6}$ is obtained, consistent with existing measurements and exceeding the precision of previous ATLAS results. Finally, an upper limit on the signal strength for the off-shell Higgs production process of 6.5 is obtained.

\subsection{Interpretation in terms of effective field theories}

New physics processes at a high energy scale $\Lambda$ that alter diboson production can be described by operators with mass dimensions larger than four in an effective field theory (EFT) framework. The dimensionless coefficients $\left(c_{i}\right)$ of the operators $O_{i}$ parameterise the strength of the coupling between new physics and SM particles, $\mathcal{L}=\mathcal{L}_{\mathrm{SM}}+\sum_{i} \frac{c_{i}}{\Lambda^{2}} O_{i}$. The higher-dimensional operators of the lowest order from purely EW processes have dimension six, and can generate aTGCs. A deviation from the SM in measured production rates or in certain kinematic distributions, as predicted by these theories, could provide evidence for BSM physics. 
For the $W W$ measurement, in the EFT framework employed, there are five dimension-six operators and the relevant EFT coefficients (coupling constants) are: $c_{W W W}, c_{W}, c_{B}, c_{\tilde{W} W W}$ and $c_{\tilde{W}}$. The distribution of the transverse momentum of the leading lepton is used to investigate aTGC parameters. No evidence for anomalous $W W Z$ and $W W \gamma$ couplings is found, hence limits on their magnitudes are set. Constraints on the EFT coefficients are determined by considering one operator at a time using the unfolded $p_{\mathrm{T}}^{\text {lead } \ell}$ fiducial cross section. Due to the higher centre-of-mass energy, the limits observed are more restrictive than those previously published by the ATLAS and CMS collaborations in the $W W$ final state. The sensitivity to dimension-six operators mostly stems from their direct effect on the $W W$ cross section as a function of $p_{\mathrm{T}}^{\text {lead } \ell}$, except for the $c_{W}$ coefficient where both the direct contribution and the interference between the SM and terms containing EFT operators contribute equally.

Since no significant deviations from the SM are observed in the $Z Z$ analysis, upper limits are placed on the aTGC parameters, which typically manifest themselves as a signal excess growing rapidly as the partonic centre-of-mass energy $\sqrt{\hat{s}}$ increases. In this analysis, aTGCs are searched for using the $p_{\mathrm{T}}^{\ell \ell}$ spectrum in the fiducial region, motivated by the fact that $p_{\mathrm{T}}^{\ell \ell}$ is correlated with $\sqrt{\hat{s}}$ and has a good experimental resolution. The contribution due to aTGCs is introduced using an effective vertex function approach, including two coupling parameters that violate $C P$ symmetry, $f_{4}^{\gamma}$ and $f_{4}^{Z}$, as well as two $C P$-conserving ones, $f_{5}^{\gamma}$ and $f_{5}^{Z}$. Furthermore, the coupling parameters are used to extract information about the dimension-eight operators of the effective field theory. Since the sensitivity to possible aTGCs lies in the high- $p_{\mathrm{T}}^{\ell \ell}$ region, only the bins with $p_{\mathrm{T}}^{\ell \ell}>150 \mathrm{GeV}$ are considered in the search. Figure $4 \mathrm{~b}$ compares the measured $p_{\mathrm{T}}^{\ell \ell}$ spectrum in this region of phase space to the SM prediction alone and to the SM prediction augmented with aTGCs corresponding to different values of the coupling parameters described above. Two-dimensional confidence intervals (CIs) for each pair of coupling parameters are derived with the other two parameters set to zero. Figure 6a presents the two-dimensional CI contours for one of the six possible pairs of aTGC coupling parameters.

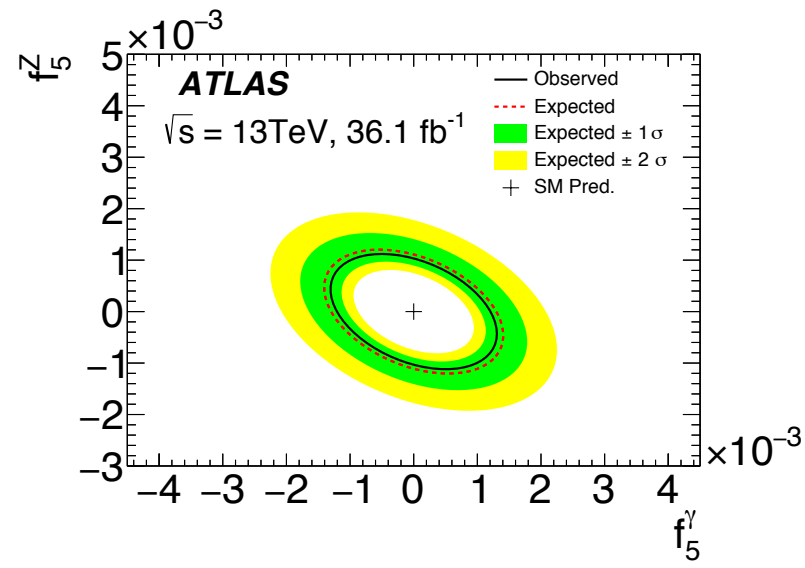

(a)

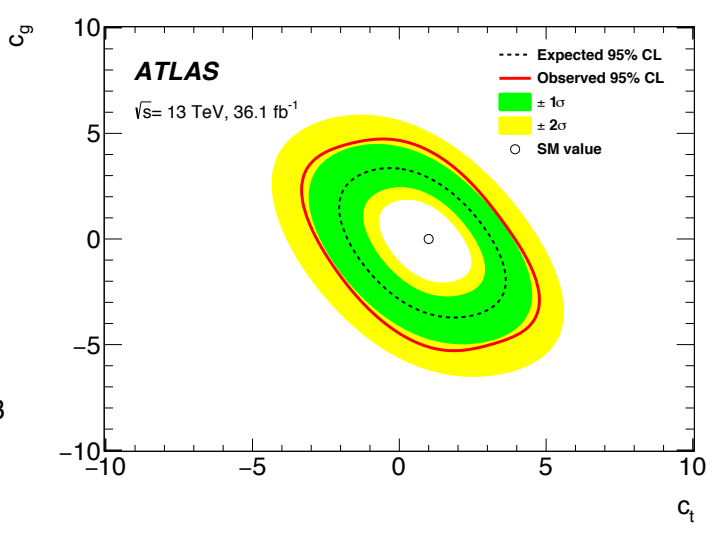

(b)

Figure 6: Observed and expected exclusion limits: (a) As function of two $f_{5}$ aTGC parameters for the $Z Z$ analysis, while the $f_{4}$ parameters are set to zero [3]. (b) In the $c_{g}$ versus $c_{t}$ plane for modified $t \bar{t} H$ and $g g H$ couplings, as obtained in the four-lepton analysis [4]. 
In the four-lepton analysis the detector-corrected four-lepton mass distribution is used to constrain possible BSM modifications of the couplings of the Higgs boson to top quarks $\left(c_{t}\right)$ and gluons $\left(c_{g}\right)$, which is predicted to be zero in the SM. On-shell rates for Higgs production via gluon-gluon fusion are only sensitive to $\left|c_{t}+c_{g}\right|^{2}$, but measurements at higher mass can be used to probe these parameters independently, as the partonic centre-of-mass energy of the process becomes larger than the top-quark mass. This provides an interesting test of the off-shell behaviour beyond dedicated measurements based on the rare $t \bar{t} H$ production mode. The yield from $g g \rightarrow 4 \ell$ is parameterised as a function of $c_{t}$ and $c_{g}$. The observed and expected exclusion contours are shown in Figure 6b, where the expected limit has green and yellow bands indicating uncertainties of $1 \sigma$ and $2 \sigma$. The parameter space which lies outside of the observed contour is excluded.

\section{Measurements of three massive vector bosons using 2015-2017 data}

\subsection{Evidence for $V V V$ production}

The joint production of three vector bosons is a rare process in the Standard Model. Studies of triboson production can test the non-Abelian gauge structure of the SM theory and any deviations from the SM prediction would provide hints of new physics at higher energy scales. Triboson production has been studied at the LHC using data taken at $\sqrt{s}=8 \mathrm{TeV}$ for processes such as $\gamma \gamma \gamma$, $W \gamma \gamma, Z \gamma \gamma, W W \gamma, W Z \gamma$ and $W W W$.

A search for the production of three massive vector bosons is performed, extracting one common signal modifier from a simultaneous fit to four selection regions targeting different channels. Events with two same-sign leptons and at least two reconstructed jets are selected to search for $W W W \rightarrow \ell v \ell v q q$. Events with three leptons without any SFOS lepton pairs are used to search for $W W W \rightarrow \ell v \ell v \ell v$, while events with three leptons and at least one SFOS lepton pair and one or more reconstructed jets are used to search for $W W Z \rightarrow \ell v q q \ell \ell$. Finally, events with four leptons are analysed to search for $W W Z \rightarrow \ell v \ell v \ell \ell$ and $W Z Z \rightarrow q q \ell \ell \ell \ell$.

At LO in QCD, the production of three massive vector bosons can proceed via the radiation of each vector boson from a fermion, from an associated boson production with an intermediate boson $\left(W, Z / \gamma^{*}\right.$ or $H$ ) decaying into two vector bosons, or from a QGC vertex. Representative Feynman diagrams are shown in Figure 7.
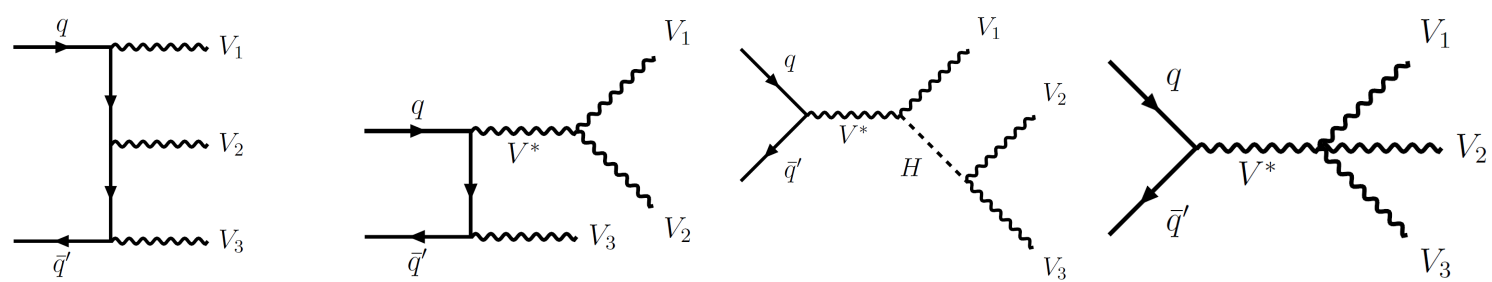

Figure 7: Representative Feynman diagrams at LO for the production of three massive vector bosons, including diagrams sensitive to triple and quartic gauge couplings.

Triboson signal events were generated using SHERPA where all three bosons are on-mass-shell. Events with off-mass-shell bosons through $W H \rightarrow W V V^{*}$ and $Z H \rightarrow Z V V^{*}$ were generated using PowhEG-Box or PythiA. Both on-mass-shell and off-mass-shell processes were generated at NLO 
QCD accuracy and are included in the signal definition. The expected cross sections for $W W W$ and $W W Z$ production are $0.50 \mathrm{pb}$ and $0.29 \mathrm{pb}$, respectively, with an uncertainty of $\sim 10 \%$.

In order to reject leptons likely to be originating from heavy-flavour decays, "nominal" leptons have to pass a requirement on a dedicated boosted decision tree (BDT). Electrons have to pass an additional BDT requirement to reject electrons likely to have the electric charge wrongly measured.

To select $\ell v \ell v q q$ candidates, events are required to have exactly two nominal leptons with the same electric charge, at least two jets, and no identified $b$-tagged jets. Four regions are considered, based on the lepton flavour, namely $e e, e \mu, \mu e$, and $\mu \mu$. Requirements on the invariant mass of the dilepton system are imposed, in order to reduce the contribution from the $W Z$ process. Cuts applied on the dijet invariant mass and $\Delta \eta_{j j}$ mainly reduce the contributions from the same-sign $W W$ vector boson scattering process. Additionally, in the ee final state, requirements on $E_{\mathrm{T}}^{\text {miss }}$ and $m_{\ell \ell}$ are imposed to reduce contamination from $Z \rightarrow e e$ where the charge of one electron is misidentified. To select $\ell v \ell v \ell v$ candidates, events are required to have exactly three nominal leptons and no identified $b$-tagged jets. To reduce the contribution from the $W Z$ process, events are required to have no SFOS lepton pairs, and thus only $\mu^{ \pm} e^{\mp} e^{\mp}$ and $e^{ \pm} \mu^{\mp} \mu^{\mp}$ events are selected.

A major background originates from the $W Z+$ jets $\rightarrow \ell v \ell \ell+$ jets process, contributing to the $\ell v \ell v q q$ channel when one lepton is not reconstructed or identified, or to the $\ell v \ell v \ell v$ channel, when a $Z$ boson decays into a pair of $\tau$ leptons both of which decay to an electron or muon. Simulation (SHERPA) is used to estimate this background. Contributions from SM processes that produce at least one non-prompt lepton are estimated using a data-driven method by introducing "fake" leptons. Fake leptons are defined by looser requirements and are mutually exclusive with the nominal leptons. Simulation shows that the $t \bar{t}$ process is the dominant contributor of events with fake leptons. Events containing one (two) nominal lepton(s) and one fake lepton are scaled by a "fake factor" to predict the non-prompt lepton background contribution in the $\ell v \ell v q q$ ( $\ell v \ell v \ell v)$ channel. The fake factor is derived from two $t \bar{t}$-enriched regions selected with two or three leptons (no SFOS lepton pairs) and exactly one $b$-tagged jet. Events resulting from the $V \gamma j j$ production can pass the signal selection criteria if the photon is misreconstructed as an electron. This contribution is evaluated using a data-driven method similar to the non-prompt lepton background evaluation by introducing "photon-like" electrons. The charge misidentification background originates from processes that produce oppositely-charged prompt leptons where one lepton's charge is misidentified and results in final states with two same-sign leptons. The background is estimated using a data-driven technique.

The experimental signature of the $W V Z \rightarrow \ell v q q \ell \ell, W W Z \rightarrow \ell v \ell v \ell \ell$, and $W Z Z \rightarrow q q \ell \ell \ell \ell$ processes is the presence of three or four leptons. In order to increase the signal acceptance, a looser lepton definition is also used. Six regions are defined with either three or four loose leptons, sensitive to triboson final states containing $Z$ bosons. Among all possible SFOS lepton pairs, the one with $m_{\ell \ell}$ closest to $m_{Z}$ is defined as the $Z$ candidate. In all regions, the presence of such a $Z$ candidate with $\left|m_{\ell \ell}-m_{Z}\right|<10 \mathrm{GeV}$, is required. Furthermore, any SFOS lepton pair combination is required to have a minimum invariant mass of $m_{\ell \ell}>12 \mathrm{GeV}$. Events with $b$-tagged jets are vetoed. For the three-lepton channel, the lepton which is not part of the $Z$ candidate is required to be a nominal lepton and the scalar sum of the transverse momenta of all leptons and jets is required to be large, significantly reducing the contribution of the $Z \rightarrow \ell \ell$ processes with one additional non-prompt lepton. Three regions are defined according to the number of jets in the event: one jet 
$(3 \ell-1 \mathrm{j})$, two jets $(3 \ell-2 \mathrm{j})$, and at least three jets $(3 \ell-3 \mathrm{j})$. For the four-lepton channel, the third and fourth leading leptons are required to be nominal leptons, and the two leptons which are not part of the $Z$ candidate definition are required to have opposite charges. They are used to define three regions, depending on whether they are different-flavour ( $4 \ell$-DF), or same-flavour and their mass is compatible with the $Z$ boson mass ( $4 \ell$-SF-Z) or not ( $4 \ell$-SF-noZ).

In each of the six regions, the distribution of a dedicated BDT discriminant, separating the $W V Z$ signal from the dominating diboson background, is fed as input to the binned maximumlikelihood fit to extract the signal. For the three-lepton channels, 12-15 input variables are used. The variables are chosen from a list of discriminating variables, including the trilepton invariant mass, the invariant mass of different lepton or jet pairs, the leptons' and jets' $p_{\mathrm{T}}$, the number of reconstructed jets, the scalar sum of all leptons' or jets' $p_{\mathrm{T}}, E_{\mathrm{T}}^{\mathrm{miss}}, H_{\mathrm{T}}$ and the invariant mass of all leptons, jets and $E_{\mathrm{T}}^{\mathrm{miss}}$. For the four-lepton channels, six input variables are used for each of the final states. These variables are chosen from the following list: $E_{\mathrm{T}}^{\text {miss }}, H_{\mathrm{T}}$, the scalar sum of all leptons' $p_{\mathrm{T}}$, the invariant masses of lepton pairs, the four-lepton invariant mass, the number of reconstructed jets, and the scalar sum of all jets' $p_{\mathrm{T}}$.

Due to the required presence of nominal leptons in the three- and four-lepton channels, backgrounds with a $Z$ boson and non-prompt leptons are reduced. The remaining backgrounds are dominated by processes with prompt leptons and thus the backgrounds are estimated using simulation. The $t \bar{t} Z$ background is determined in a region defined like the $3 \ell-3 \mathrm{j}$ region with the exception that no requirement on $H_{\mathrm{T}}$ is applied, and at least four jets are required, of which at least two are $b$-tagged. This region is included as a single-bin control region in the fit model.

The $W W W, W W Z$ and $W Z Z$ regions are combined using a binned profile likelihood method based on a simultaneous fit to distributions in the eleven signal regions $(e e, e \mu, \mu e, \mu \mu, \ell v \ell v \ell v$, $3 \ell-1 \mathrm{j}, 3 \ell-2 \mathrm{j}, 3 \ell-3 \mathrm{j}, 4 \ell-\mathrm{DF}, 4 \ell$-SF-Z, and $4 \ell$-SF-noZ) and the $t \bar{t} Z$ background control region. The distributions used in the fit are the $m_{j j}$ distributions for the $\ell v \ell v q q$ channel and the full BDT distributions for the $W V Z$ three-lepton and four-lepton channels. The number of selected events in the $\ell v \ell v \ell v$ channel and the $t \bar{t} Z$ control region are each included as a single bin in the fit. In total, 186 bins are used in the combined fit. The likelihood function depends on the signal-strength parameter $\mu$, a multiplicative factor that scales the number of expected signal events, and a set of nuisance parameters that encode the effect of systematic uncertainties of the signal and background expectations, implemented as Gaussian, log-normal or Poisson constraints. The same value for $\mu$ is assumed for the on- and off-mass-shell $W W W, W W Z$ and $W Z Z$ processes.

Figure 8 shows the comparison between data and post-fit prediction of the combined $m_{j j}$ distribution for the $\ell v \ell v q q$ channel, and the BDT output distributions in the $3 \ell-2 \mathrm{j}$ and $4 \ell$-DF regions for the $W V Z$ analysis. The $3 \ell-2 \mathrm{j}$ and $4 \ell$-DF regions are chosen since they have the best sensitivity among the three-lepton and four-lepton channels. Data and predictions agree in all distributions.

Figure 9a shows the observed value of the signal strength. Results are shown for the $W W W$ and $W V Z$ channels separately, fixing the other signal to its SM expectation, and combined. The combined best-fit signal strength for the $V V V$ process, obtained by the fit to the eleven signal regions and one control region is $1.38_{-0.37}^{+0.39}$ with respect to the SM prediction. The statistical uncertainty is ${ }_{-0.24}^{+0.25}$ and the systematic uncertainty is ${ }_{-0.27}^{+0.30}$. The largest systematic uncertainties come from uncertainties related to data-driven background evaluations affecting the $W W W$ channels and from theoretical uncertainties related to renormalisation and factorisation scale variations, mostly 


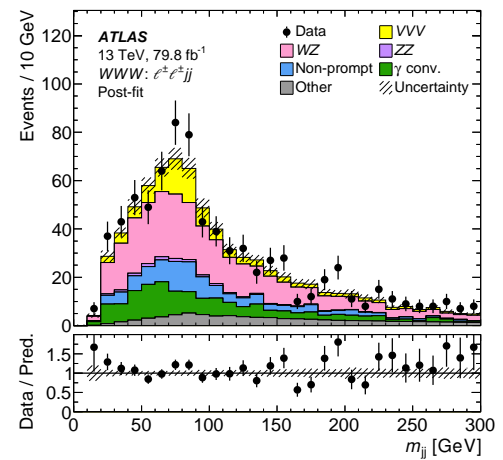

(a)

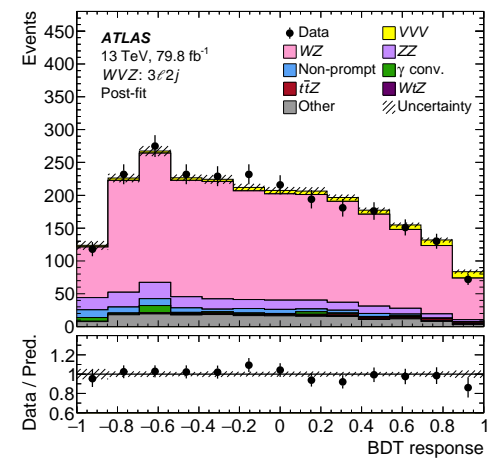

(b)

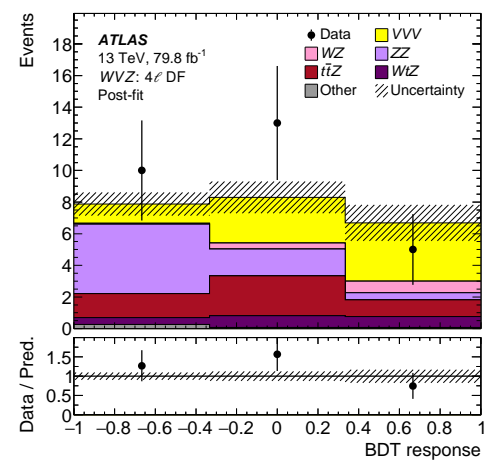

(c)

Figure 8: Post-fit distributions [5] of (a) $m_{j j}$ for the $W W W \rightarrow \ell v \ell v q q$ analysis (ee, $e \mu, \mu e, \mu \mu$ combined), and of the BDT output for the (b) $3 \ell-2 \mathrm{j}$ and (c) $4 \ell$-DF channels of the $W V Z$ analysis.

in the diboson background, evaluated using simulations. The observed (expected) significance for $W W W$ production is $3.3 \sigma(2.4 \sigma)$, and $2.9 \sigma(2.0 \sigma)$ for $W V Z$ production. The overall observed (expected) significance for $V V V$ production is $4.0 \sigma(3.1 \sigma)$, constituting evidence for the production of three massive vector bosons. Figure $9 \mathrm{~b}$ shows the data, background and signal yields, where the final-discriminant bins in all signal regions are combined into bins of $\log _{10}(\mathrm{~S} / \mathrm{B}), \mathrm{S}$ being the expected signal yield and B the background yield. The background and signal yields are shown after the global signal-plus-background fit to the data.

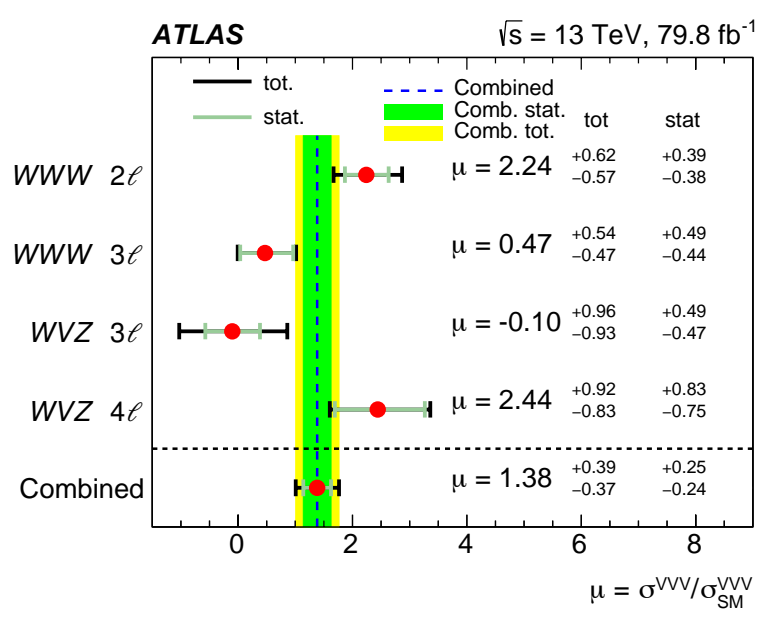

(a)

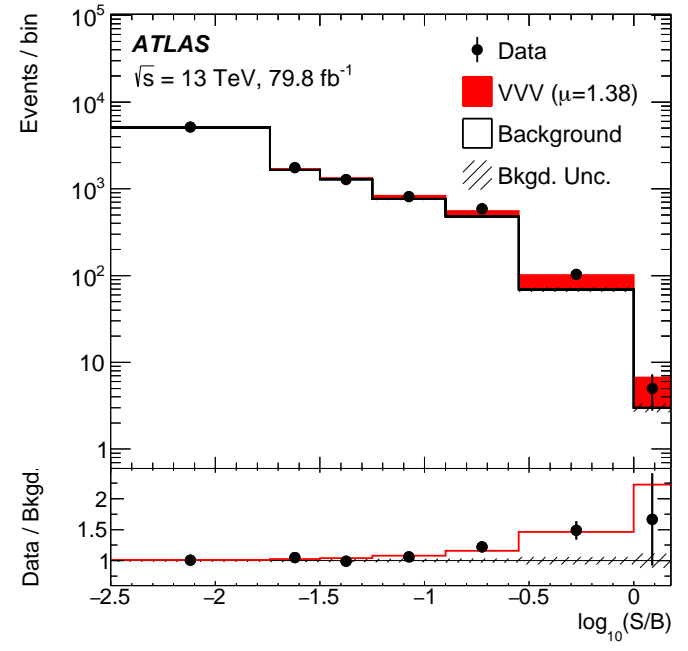

(b)

Figure 9: (a) Observed values of the signal strength per $V V V$ analysis channel and combination [5]. (b) All signal regions combined into bins of $\log _{10}(\mathrm{~S} / \mathrm{B})[5]$.

The measured signal strengths from the fits and their uncertainties are converted to inclusive cross-section measurements using the signal samples and the central values of the theoretical predictions. All uncertainties determined in the fit are included in the conversion, except for the nor- 
malisation uncertainty in the signal prediction. The results are: $\sigma_{W W W}=0.68_{-0.15}^{+0.16}$ (stat.) ${ }_{-0.15}^{+0.16}$ (syst.) $\mathrm{pb}$ and $\sigma_{W W Z}=0.49 \pm 0.14$ (stat. $)_{-0.13}^{+0.14}$ (syst.) pb, in agreement with the Standard Model predictions. For the $\sigma_{W W Z}$ extraction, the $W Z Z$ normalisation is fixed to the SM expectation.

\section{Summary}

Five recent ATLAS measurements on multiboson production in $p p$ collisions at the LHC have been presented. For the four improved measurements on massive diboson final states, $W W, W Z$, $Z Z$, and four leptons, using 2015-2016 data, the measured fiducial inclusive cross sections agree with SM calculations at NNLO in QCD, including EW corrections. The total uncertainties of the measured cross sections are $4.5 \%(\mathrm{WZ}), 6.8 \%(\mathrm{ZZ}), 7.1 \%(\mathrm{WW})$ and $8.6 \%$ for the rare $Z \rightarrow$ $4 \ell$ decay. Differential distributions are compared with several calculations and generators and measurements agree in general, with some few exceptions. Sensitive distributions are used to constrain aTGCs in the framework of EFTs, improving on existing limits. In the $W Z$ channel, the helicity states of the bosons are extracted for the first time at a hadron collider. Using 2015-2017 data, ATLAS also established first evidence for the production of three massive vector boson with a combined significance of $4.0 \sigma$ and total cross-section measurements for $W W W$ and $W W Z$ with uncertainties of the order $30-40 \%$.

\section{Acknowledgements}

The author would like to thank (in alphabetical order) Ulla Blumenschein, Vadim Kostyukhin, Bogdan Malaescu, Joany Manjarrés Ramos, Matthias Schott, Andrea Sciandra, Ismet Siral, Alex Tuna and Junjie Zhu in their various roles for making the first evidence for three massive vector boson production possible.

This work was partially funded by the European Research Council under the European Union's Seventh Framework Programme ERC Consolidator Grant Agreement n. 617185 (TopCoup) and by the German Federal Ministry of Education and Research (BMBF) in FSP-103 under grant n. 05H15PDCAA.

\section{References}

[1] ATLAS Collaboration, Measurement of fiducial and differential $W^{+} W^{-}$production cross-sections at $\sqrt{s}=13 \mathrm{TeV}$ with the ATLAS detector, submitted to Eur. Phys. J. C, arXiv:1905.04242 [hep-ex].

[2] ATLAS Collaboration, Measurement of $W^{ \pm} Z$ production cross sections and gauge boson polarisation in pp collisions at $\sqrt{s}=13 \mathrm{TeV}$ with the ATLAS detector, accepted for publication in Eur. Phys. J. C, arXiv:1902.05759 [hep-ex].

[3] ATLAS Collaboration, Measurement of ZZ production in the $\ell \ell v v$ final state with the ATLAS detector in pp collisions at $\sqrt{s}=13 \mathrm{TeV}$, submitted to JHEP, arXiv:1905.07163 [hep-ex].

[4] ATLAS Collaboration, Measurement of the four-lepton invariant mass spectrum in $13 \mathrm{TeV}$ proton-proton collisions with the ATLAS detector, JHEP 04 (2019) 048, arXiv:1902.05892 [hep-ex].

[5] ATLAS Collaboration, Evidence for the production of three massive vector bosons with the ATLAS detector, submitted to Phys. Lett. B, arXiv:1903.10415 [hep-ex]. 